\title{
Matter-screened Casimir force and Casimir-Polder force in planar structures
}

\author{
Christian Raabe and Dirk-Gunnar Welsch \\ Theoretisch-Physikalisches Institut, Friedrich-Schiller-Universität Jena, \\ Max-Wien-Platz 1, D-07743 Jena, Germany \\ E-mail: C.Raabe@tpi.uni-jena.de, D.-G.Welsch@tpi.uni-jena.de
}

\begin{abstract}
Using a recently developed theory of the Casimir force (Raabe C and Welsch D-G 2005 Phys. Rev. A 71 013814), we calculate the force that acts on a plate in front of a planar wall and the force that acts on the plate in the case where the plate is part of matter that fills the space in front of the wall. We show that in the limit of a dielectric plate whose permittivity is close to unity, the force obtained in the former case reduces to the ordinary, i.e., unscreened Casimir-Polder force acting on isolated atoms. In the latter case, the theory yields the Casimir-Polder force that is screened by the surrounding matter.
\end{abstract}

Keywords: Casimir force, Casimir-Polder force, QED vacuum effects, screening effect 


\section{Introduction}

From the point of view of statistical physics, the classical electromagnetic vacuum can be characterized by the condition that all moments of the electric and induction fields vanish identically, which implies the absence of any interaction with matter. In quantum electrodynamics, this definition is precluded by the non-commutativity of canonically conjugate field quantities; thus non-vanishing moments must inevitably occur. The quantum electromagnetic vacuum can be merely characterized as the state in which all normally ordered field moments vanish identically. Clearly, the anti-normally (or otherwise non-normally) ordered field moments then cannot do so, due to virtual photon creation and destruction - a signature of the noise of the quantum vacuum. Since the electromagnetic vacuum cannot be switched off, its interaction with atomic systems cannot be switched off either, thereby giving rise to a number of observable effects. Both virtual and real photons can be involved in the interaction. Whereas the interaction of ground-state atoms with the electromagnetic vacuum takes place via virtual photon creation and destruction, the creation of real photons always requires excited atoms.

A typical example of the interaction via virtual photons is the attractive van der Waals force between two unpolarized ground-state atoms, which can be regarded as the force between electric dipoles that are induced by the fluctuating vacuum field. In the non-retarded (i.e., short-distance) limit, the potential associated with the force was first calculated by London $[1,2]$. The theory was later extended by Casimir and Polder [3] to allow for larger separations, where retardation effects cannot be disregarded. Forces which are mediated by the electromagnetic vacuum are not only observed on a microscopic level but also on macroscopic levels. Typical examples are the force that an (unpolarized) atom experiences in the presence of macroscopic (unpolarized) bodies referred to as Casimir-Polder (CP) force in the following - or the Casimir force between macroscopic (unpolarized) bodies (for a review, see, for example, [4]).

Since macroscopic bodies consist of a huge number of atoms, both the CP force and the Casimir force can be regarded as macroscopic manifestations of microscopic van der

Waals forces, and both types of forces are intimately related to each other. Clearly, they cannot be obtained, in general, from a simple superposition of two-atom van der Waals forces because such a procedure would completely ignore the interaction between the constituent atoms of the bodies, and thus also their collective influence on the structure of the body-assisted electromagnetic field [5]. The aim of the present paper is to study this problem in more detail, with special emphasis on the CP force in planar structures.

In principle, it is certainly possible to calculate $\mathrm{CP}$ and Casimir forces within the framework of microscopic quantum electrodynamics, by solving the respective manyparticle problem in some approximation. Alternatively, one can start from a macroscopic description of the bodies in terms of boundary conditions or, more generally, in terms of polarization and magnetization fields together with (phenomenologically introduced) constitutive relations. The latter, very powerful approach will be used throughout this paper. To be more specific, we will apply the theory recently developed in [6], which 
renders it possible not only to calculate the Casimir force that acts on bodies separated by empty space, but also the one which acts on bodies the interspace between which is filled with matter. As we will see, the formula for the Casimir force obtained in this way contains, as a special case, the well-known formula for the CP force acting on isolated atoms. Moreover, it can also be used to calculate the CP force acting on atoms that are constituents of matter, where the neighbouring atoms give rise to a screening effect that diminishes the force. Throughout the paper systems that are at rest are considered, which implies that the electromagnetic vacuum forces must be thought of as being balanced by some other forces.

The paper is organized as follows. After a review in section 2 of the CP force, the theory of the Casimir force as developed in [6] is outlined in section 3, and the Casimir stress in planar structures is given. Relations between the Casimir force and the unscreened as well as the screened CP force are studied in section 4 , and the results are discussed and summarized in section 5 .

\section{Casimir-Polder force}

Provided that the broadening of the atomic levels can be neglected, the CP force is conservative, i.e., expressible as the (negative) gradient of a potential - the CP potential. While this approximation may be invalid for atoms prepared in an excited state, it is well justified for ground-state atoms, in which case the CP potential can be written in the form of

$$
V^{(\mathrm{at})}(\mathbf{r})=\frac{\hbar \mu_{0}}{2 \pi} \int_{0}^{\infty} \mathrm{d} \xi \xi^{2} \alpha(i \xi) \operatorname{Tr} \mathrm{G}^{(\mathrm{S})}(\mathbf{r}, \mathbf{r}, i \xi)
$$

(for derivations, see, for example, [7, 8, 9, 10, 11]). Here, $\mathbf{r}$ is the position of the atom, $\alpha(i \xi)$ is its (ground-state) polarizability and $\mathrm{G}^{(\mathrm{S})}\left(\mathbf{r}, \mathbf{r}^{\prime}, i \xi\right)$ is the scattering part of the classical retarded Green tensor $\mathrm{G}\left(\mathbf{r}, \mathbf{r}^{\prime}, i \xi\right)$ on the imaginary frequency axis. Note that the Green tensor takes the presence of arbitrary (locally responding) magnetodielectric bodies into account within the framework of macroscopic linear electrodynamics in causal media. Only the scattering part of the Green tensor figures in equation (1); its bulk part, despite being divergent in the coincidence limit $\mathbf{r}^{\prime} \rightarrow \mathbf{r}$, does not contribute to the force on the atom and can be thus discarded from equation (1). The potential (1), which is valid to first order in $\alpha(i \xi)$, can be derived using the Green tensor scheme of electromagnetic field quantization [12] and treating the atom-field interaction in the electric dipole approximation and the lowest (non-vanishing) order of perturbation theory.

In particular, for a ground-state atom in front of a planar magnetodielectric wall, equation (1) leads to (see, for example, $[7,8,9,13,14]$ )

$$
V^{(\mathrm{at})}(z)=\frac{\hbar \mu_{0}}{8 \pi^{2}} \int_{0}^{\infty} \mathrm{d} \xi \xi^{2} \alpha(i \xi) \int_{0}^{\infty} \mathrm{d} q \frac{q}{\kappa} e^{-2 \kappa z}\left[r_{1-}^{s}-r_{1-}^{p}\left(1+\frac{2 q^{2}}{\xi^{2} / c^{2}}\right)\right]
$$

$\left(\kappa^{2}=\xi^{2} / c^{2}+q^{2}\right)$, where the atom is situated at some position $z>0$, and the magnetodielectric wall (which may have a (1D) internal structure, for example, a 
planarly layered one) extends from some negative $z$-value up to $z=0$. Note that the effect of the wall is fully described in terms of the (generalized) reflection coefficients $r_{1-}^{s}$ and $r_{1-}^{p}$, both of which are functions of the imaginary frequency $i \xi$ and the transverse wave vector projection $q$ ( $s, p$, polarization indices).

Two comments on equations (1) and (2) seem to be advisable. First, the neglect of level broadening might suggest that $\alpha(\omega)$ has poles on the real frequency axis, such as

$$
\alpha(\omega) \sim \sum_{k} \frac{\Omega_{k}^{2}}{\omega_{k}^{2}-\omega^{2}} .
$$

In fact, equation (3) has to be understood as

$$
\alpha(\omega) \sim \lim _{\gamma \rightarrow 0+} \sum_{k} \frac{\Omega_{k}^{2}}{\omega_{k}^{2}-\omega^{2}-i \gamma \omega},
$$

where the limit prescription $\gamma \rightarrow 0+$ reminds one of the proper response function properties [15] of $\alpha(\omega)$. Throughout this paper it will not be necessary to make use of any particular form of the polarizability. Second, although the (magnetodielectric) bodies can be quite arbitrary, it is important that the atom under study is an isolated one, i.e., equations (1) and (2) do not apply to atoms in matter. Needless to say that a convincing consideration of atoms in matter must include local field corrections. Correspondingly, the reflection coefficients in equation (2) refer to the reflection of waves being incident on the wall from free space.

\section{Casimir force}

Let us now consider the Casimir force acting on a macroscopic body in the presence of other bodies. In the zero-temperature limit, it is just the ground-state expectation value of the Lorentz force acting on the charges and currents which constitute the body on the level of macroscopic electrodynamics, i.e., the charge density $\hat{\rho}(\mathbf{r})$ can be given by

$$
\hat{\rho}(\mathbf{r})=\int_{0}^{\infty} \mathrm{d} \omega \underline{\hat{\rho}}(\mathbf{r}, \omega)+\text { H. c. }
$$

and the current density $\hat{\mathbf{j}}(\mathbf{r})$ accordingly, with

$$
\underline{\hat{\rho}}(\mathbf{r}, \omega)=-\varepsilon_{0} \boldsymbol{\nabla} \cdot\{[\varepsilon(\mathbf{r}, \omega)-1] \underline{\hat{\mathbf{E}}}(\mathbf{r}, \omega)\}+(i \omega)^{-1} \boldsymbol{\nabla} \cdot \underline{\hat{\mathbf{j}}}_{\mathrm{N}}(\mathbf{r}, \omega)
$$

and

$\underline{\hat{\mathbf{j}}}(\mathbf{r}, \omega)=-i \omega \varepsilon_{0}[\varepsilon(\mathbf{r}, \omega)-1] \underline{\hat{\mathbf{E}}}(\mathbf{r}, \omega)+\nabla \times\left\{\mu_{0}^{-1}\left[1-\mu^{-1}(\mathbf{r}, \omega)\right] \underline{\hat{\mathbf{B}}}(\mathbf{r}, \omega)\right\}+\underline{\hat{\mathbf{j}}}_{\mathrm{N}}(\mathbf{r}, \omega)$

(for details, see [6]). Here, $\hat{\mathbf{j}}_{\mathrm{N}}(\mathbf{r}, \omega)$ is the current density that acts as a Langevin noise source in the operator Maxwell equations, $\varepsilon(\mathbf{r}, \omega)$ and $\mu(\mathbf{r}, \omega)$ are the permittivity and permeability, respectively, and $\underline{\hat{\mathbf{E}}}(\mathbf{r}, \omega)$ and $\underline{\hat{\mathbf{B}}}(\mathbf{r}, \omega)$ are the (positive) frequency parts of the electric field and the induction field, respectively,

$$
\underline{\hat{\mathbf{E}}}(\mathbf{r}, \omega)=i \mu_{0} \omega \int \mathrm{d}^{3} r^{\prime} \mathrm{G}\left(\mathbf{r}, \mathbf{r}^{\prime}, \omega\right) \cdot \hat{\mathbf{j}}_{\mathrm{N}}\left(\mathbf{r}^{\prime}, \omega\right)
$$


Matter-screened Casimir force and Casimir-Polder force

$$
\underline{\hat{\mathbf{B}}}(\mathbf{r}, \omega)=\mu_{0} \nabla \times \int \mathrm{d}^{3} r^{\prime} \mathrm{G}\left(\mathbf{r}, \mathbf{r}^{\prime}, \omega\right) \cdot \hat{\mathbf{j}}_{\mathrm{N}}\left(\mathbf{r}^{\prime}, \omega\right) \text {. }
$$

According to [6], the Casimir force on a magnetodielectric body of volume $V$ can then be expressed in terms of the Casimir stress as a surface integral,

$$
\mathbf{F}=\int_{\partial V} \mathrm{~d} \mathbf{a} \cdot \mathrm{T}(\mathbf{r}, \mathbf{r})
$$

where

$$
\begin{aligned}
& \mathrm{T}(\mathbf{r}, \mathbf{r})=\lim _{\mathbf{r}^{\prime} \rightarrow \mathbf{r}}\left[\theta\left(\mathbf{r}, \mathbf{r}^{\prime}\right)-\frac{1}{2} 1 \operatorname{Tr} \theta\left(\mathbf{r}, \mathbf{r}^{\prime}\right)\right] \\
& \theta\left(\mathbf{r}, \mathbf{r}^{\prime}\right)=-\frac{\hbar}{\pi} \int_{0}^{\infty} \mathrm{d} \xi\left[\frac{\xi^{2}}{c^{2}} \mathrm{G}^{(\mathrm{S})}\left(\mathbf{r}, \mathbf{r}^{\prime}, i \xi\right)+\nabla \times \mathrm{G}^{(\mathrm{S})}\left(\mathbf{r}, \mathbf{r}^{\prime}, i \xi\right) \times \overleftarrow{\nabla^{\prime}}\right]
\end{aligned}
$$

with the body under study being taken into account in the definition of the scattering Green tensor. It is worth noting that equations (11) and (12) also apply if the interspace between the bodies is not empty but also filled with magnetodielectric matter (which has to be homogeneous at least in some small neighbourhood of the body under consideration). In this case the Casimir force is expected to be diminished as compared to the case where the surrounding matter is absent, because of the screening effect of the matter.

Let us apply equation (11) (together with equation (12)) to the $j$ th (homogeneous) layer of a planar magnetodielectric multi-layer structure. For such systems the Green tensor is well known $[16,17]$, leading to $\left(0<z<d_{j} ; d_{j}\right.$, thickness of the layer $)$

$$
T_{z z}(\mathbf{r}, \mathbf{r})=\frac{\hbar}{8 \pi^{2}} \int_{0}^{\infty} \mathrm{d} \xi \int_{0}^{\infty} \mathrm{d} q q \frac{\mu_{j}(i \xi)}{i \beta_{j}(i \xi, q)} g_{j}(z, i \xi, q),
$$

where

$$
\begin{aligned}
g_{j}(z, \omega, q)= & 2\left[\beta_{j}^{2}\left(1+n_{j}^{-2}\right)-q^{2}\left(1-n_{j}^{-2}\right)\right] D_{j s}^{-1} r_{j+}^{s} r_{j-}^{s} e^{2 i \beta_{j} d_{j}} \\
& +2\left[\beta_{j}^{2}\left(1+n_{j}^{-2}\right)+q^{2}\left(1-n_{j}^{-2}\right)\right] D_{j p}^{-1} r_{j+}^{p} r_{j-}^{p} e^{2 i \beta_{j} d_{j}} \\
& -\left(\beta_{j}^{2}+q^{2}\right)\left(1-n_{j}^{-2}\right) D_{j s}^{-1}\left[r_{j-}^{s} e^{2 i \beta_{j} z}+r_{j+}^{s} e^{2 i \beta_{j}\left(d_{j}-z\right)}\right] \\
& +\left(\beta_{j}^{2}+q^{2}\right)\left(1-n_{j}^{-2}\right) D_{j p}^{-1}\left[r_{j-}^{p} e^{2 i \beta_{j} z}+r_{j+}^{p} e^{2 i \beta_{j}\left(d_{j}-z\right)}\right],
\end{aligned}
$$

with

$$
\begin{aligned}
& n_{j}^{2}=n_{j}^{2}(\omega)=\varepsilon_{j}(\omega) \mu_{j}(\omega), \\
& \beta_{j}=\beta_{j}(\omega, q)=\left(\omega^{2} n_{j}^{2} / c^{2}-q^{2}\right)^{1 / 2}, \\
& D_{j \sigma}=D_{j \sigma}(\omega, q)=1-r_{j+}^{\sigma} r_{j-}^{\sigma} e^{2 i \beta_{j} d_{j}},
\end{aligned}
$$

and $r_{j \pm}^{\sigma}=r_{j \pm}^{\sigma}(\omega, q)$ being the generalized reflection coefficients associated with the $j$ th layer $(\sigma=s, p)$. Since $-i \beta_{j}$ is purely real and nonnegative at imaginary frequencies, we will use the notation $\kappa_{j}=-i \beta_{j}(i \xi, q)$ in the remainder of the paper. 


\section{Unscreened versus screened Casimir-Polder force}

Equation (10) together with equations (11) and (12) contains the unscreened CP force that acts on isolated atoms as limiting case. Moreover, it enables one to calculate also the screened CP force acting on atoms that are constituents of matter. To illustrate this, let us consider planar systems as sketched in figure 1 and begin with the unscreened $\mathrm{CP}$ force.

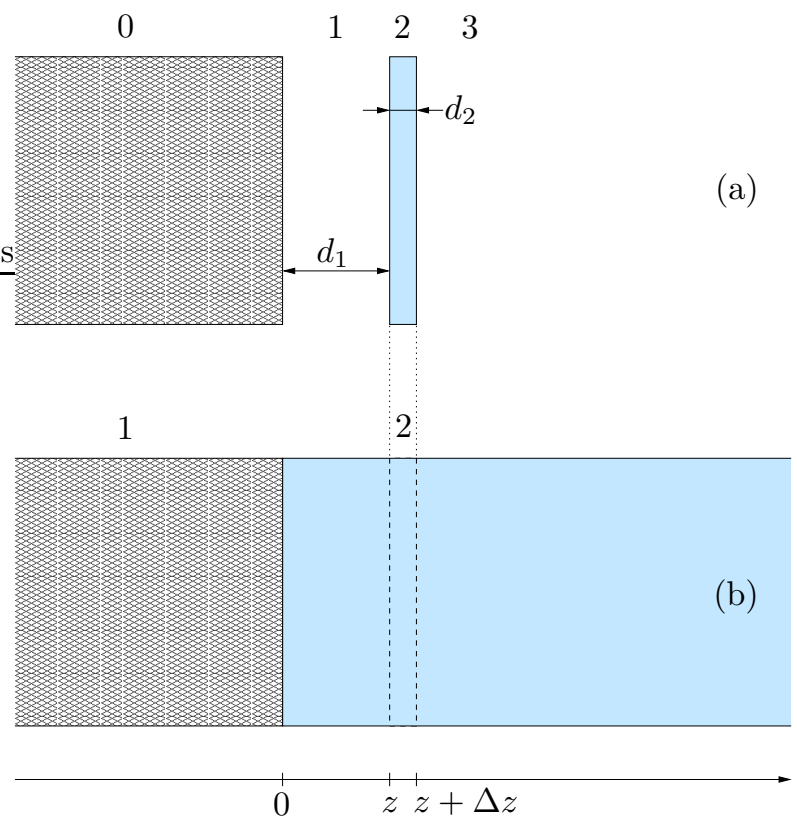

Figure 1. A homogeneous plate in front of a planar magnetodielectric wall (a); the plate is part of matter that fills the space in front of the wall (b).

(This figure is in colour only in the electronic version)

\subsection{Casimir-Polder force on isolated atoms}

For this purpose we first calculate the Casimir force acting on a (homogeneous) plate of thickness $d_{2}=\Delta z$ in front of a planar wall according to the four-layer system in figure 1 (a), with the regions 1 and 3 being empty. In this case we have to set $n_{1}=n_{3}=1$ and $d_{3} \rightarrow \infty$ (and hence $r_{3+}^{\sigma} \rightarrow 0$ ) in equations (13) and (14), which in particular implies that the stress in the empty-space region 1 is independent of position and vanishes in the semi-infinite empty-space region 3. According to equations (10) and (13), the total Casimir force (per transverse unit area) acting on the plate (layer 2) can then be written as $\left[\kappa_{1}=\kappa_{3}=\kappa=\left(\xi^{2} / c^{2}+q^{2}\right)^{1 / 2}\right]$

$$
F=\frac{\hbar}{8 \pi^{2}} \int_{0}^{\infty} \mathrm{d} \xi \int_{0}^{\infty} \mathrm{d} q \frac{q}{\kappa} g_{1}\left(d_{1}, i \xi, q\right),
$$

where

$g_{1}\left(d_{1}, i \xi, q\right)=-4 \kappa^{2} \sum_{\sigma=s, p} \frac{r_{1+}^{\sigma} r_{1-}^{\sigma} e^{-2 \kappa d_{1}}}{1-r_{1+}^{\sigma} r_{1-}^{\sigma} e^{-2 \kappa d_{1}}}=-4 \kappa^{2} \sum_{\sigma=s, p} \sum_{m=1}^{\infty}\left[r_{1+}^{\sigma} r_{1-}^{\sigma} e^{-2 \kappa d_{1}}\right]^{m}$. 
Since the reflection coefficients $r_{1-}^{\sigma}$ (containing the details of the wall structure) are independent of the properties of the plate, they need not be further specified. In the case of a homogeneous plate, the reflection coefficients $r_{1+}^{\sigma}$ read

$$
r_{1+}^{\sigma}=\frac{r_{1 / 2}^{\sigma}\left(1-e^{-2 \kappa_{2} d_{2}}\right)}{1-r_{1 / 2}^{\sigma 2} e^{-2 \kappa_{2} d_{2}}} \quad(\sigma=s, p),
$$

where $r_{1 / 2}^{\sigma}$ are the usual single-interface (Fresnel) amplitudes,

$$
r_{1 / 2}^{s}=\frac{\kappa \mu_{2}-\kappa_{2}}{\kappa \mu_{2}+\kappa_{2}}, \quad r_{1 / 2}^{p}=\frac{\kappa \varepsilon_{2}-\kappa_{2}}{\kappa \varepsilon_{2}+\kappa_{2}} .
$$

To recover the ordinary (unscreened) CP force, let us consider a nonmagnetic plate $\left(\mu_{2}=1\right)$ consisting of weakly dielectric material $\left(\left|\varepsilon_{2}-1\right| \ll 1\right)$ and expand to first order in $\varepsilon_{2}-1$. In this approximation, we may set

$$
\frac{\kappa_{2}}{\kappa}=1+\frac{2 \xi^{2}}{\kappa^{2} c^{2}}\left(\varepsilon_{2}-1\right)
$$

and equations (21) approximate to

$$
\begin{aligned}
& r_{1 / 2}^{s}=-\left(\varepsilon_{2}-1\right) \frac{\xi^{2}}{4 \kappa^{2} c^{2}}, \\
& r_{1 / 2}^{p}=\frac{\varepsilon_{2}-1}{2}\left(1-\frac{\xi^{2}}{2 \kappa^{2} c^{2}}\right),
\end{aligned}
$$

so that equations (20) read

$$
\begin{aligned}
& r_{1+}^{s}=-\left(\varepsilon_{2}-1\right) \frac{\xi^{2}}{4 \kappa^{2} c^{2}}\left(1-e^{-2 \kappa \Delta z}\right), \\
& r_{1+}^{p}=\frac{\varepsilon_{2}-1}{2}\left(1-\frac{\xi^{2}}{2 \kappa^{2} c^{2}}\right)\left(1-e^{-2 \kappa \Delta z}\right) .
\end{aligned}
$$

Inserting equations (25) and (26) in equation (19), we see that $g_{1}\left(d_{1}, i \xi, q\right)$ approximates to

$$
\begin{aligned}
g_{1}\left(d_{1}, i \xi, q\right) & =-4 \kappa^{2} \sum_{\sigma=s, p} r_{1+}^{\sigma} r_{1-}^{\sigma} e^{-2 \kappa d_{1}} \\
& =\left(\varepsilon_{2}-1\right) \kappa^{2} e^{-2 \kappa d_{1}}\left(1-e^{-2 \kappa \Delta z}\right)\left[r_{1-}^{s}\left(1-\frac{q^{2}}{\kappa^{2}}\right)-r_{1-}^{p}\left(1+\frac{q^{2}}{\kappa^{2}}\right)\right] .
\end{aligned}
$$

Note that it solely results from the $m=1$ ('single round-trip') term (in the second, expanded form of equation (19)). Substitution of equation (27) into equation (18) yields the Casimir force (per transverse unit area) to first order in $\varepsilon_{2}-1$ :

$$
\begin{aligned}
F=\frac{\hbar}{8 \pi^{2}} \int_{0}^{\infty} & \mathrm{d} \xi\left(\varepsilon_{2}-1\right) \\
& \times \int_{0}^{\infty} \mathrm{d} q q \kappa e^{-2 \kappa d_{1}}\left(1-e^{-2 \kappa \Delta z}\right)\left[r_{1-}^{s}\left(1-\frac{q^{2}}{\kappa^{2}}\right)-r_{1-}^{p}\left(1+\frac{q^{2}}{\kappa^{2}}\right)\right] .
\end{aligned}
$$

It is not difficult to prove that equation (28) can be rewritten as

$$
F=\int_{d_{1}}^{d_{1}+\Delta z} \mathrm{~d} z f(z),
$$


where the force density $f(z)$ can be derived from a potential $V(z)$ as follows:

$$
\begin{gathered}
f(z)=-\frac{\partial V(z)}{\partial z} \\
V(z)=\frac{\hbar}{8 \pi^{2}} \int_{0}^{\infty} \mathrm{d} \xi\left(\varepsilon_{2}-1\right) \int_{0}^{\infty} \mathrm{d} q q \kappa e^{-2 \kappa z}\left[r_{1-}^{s}\left(1-\frac{q^{2}}{\kappa^{2}}\right)-r_{1-}^{p}\left(1+\frac{q^{2}}{\kappa^{2}}\right)\right] .
\end{gathered}
$$

Let us suppose that the plate consists of atom-like basic constituents of polarizability $\alpha(i \xi)$ and $\eta$ is the (constant) number density of the atoms. Using the relation

$$
\varepsilon_{2}(i \xi)-1=\frac{\eta}{\varepsilon_{0}} \alpha(i \xi)
$$

which is valid in the case of weakly dielectric material, from inspection of equations (29)-(31) we see that

$$
F^{(\text {at })}(z)=\eta^{-1} f(z)
$$

can be regarded as the force acting on an atom at position $z$ in the plate, where the associated potential reads

$V^{(\mathrm{at})}(z)=\frac{\hbar}{8 \pi^{2} \varepsilon_{0}} \int_{0}^{\infty} \mathrm{d} \xi \alpha(i \xi) \int_{0}^{\infty} \mathrm{d} q q \kappa e^{-2 \kappa z}\left[r_{1-}^{s}\left(1-\frac{q^{2}}{\kappa^{2}}\right)-r_{1-}^{p}\left(1+\frac{q^{2}}{\kappa^{2}}\right)\right]$.

It is straightforwardly checked that equation (34) is identical with equation (2), i.e., with the standard CP potential. Hence, $F^{(a t)}(z)$ is nothing but the unscreened CP force that acts on a single (ground-state) atom at position $z$ in front of the wall. Clearly, this interpretation presupposes that $\alpha(i \xi)$ is really the polarizability of a single atom. Otherwise $\alpha(i \xi)$ and $\eta$ are rather formal quantities defined by equation (32), so that the introduction of $F^{(a t)}(z)$ is also rather formal. Although equations (33) and (34) are of course correct, equations (30) and (31) may be more appropriate in this case.

\subsection{Screened Casimir-Polder force on medium atoms}

Let us now consider the case where the plate is part of matter that fills the space in front of the wall according to the two-layer system in figure 1(b). In this case from equation (14) it follows that $\left(d_{2} \rightarrow \infty\right.$ and hence $\left.r_{2+}^{\sigma}=0\right)$

$g_{2}(z+\Delta z, \omega, q)-g_{2}(z, \omega, q)=e^{-2 \kappa_{2} z}\left(q^{2}-\kappa_{2}^{2}\right)\left(1-n_{2}^{-2}\right)\left(r_{2-}^{p}-r_{2-}^{s}\right)\left(e^{-2 \kappa_{2} \Delta z}-1\right)$,

so that, according to equations (10) and (13) the force (per transverse unit area) on the plate reads

$$
F=\frac{\hbar}{8 \pi^{2}} \int_{0}^{\infty} \mathrm{d} \xi \frac{\xi^{2}}{c^{2}} \mu_{2}\left(n_{2}^{2}-1\right) \int_{0}^{\infty} \mathrm{d} q \frac{q}{\kappa_{2}} e^{-2 \kappa_{2} z}\left(r_{2-}^{p}-r_{2-}^{s}\right)\left(e^{-2 \kappa_{2} \Delta z}-1\right) .(36
$$

Now we again focus on nonmagnetic and weakly dielectric matter, i.e., $\mu_{2}=1$, $\left|\varepsilon_{2}-1\right| \ll 1$. It is not difficult to see that to first order in $\varepsilon_{2}-1$ equation (36) yields

$$
F=\frac{\hbar}{8 \pi^{2}} \int_{0}^{\infty} \mathrm{d} \xi \frac{\xi^{2}}{c^{2}}\left(\varepsilon_{2}-1\right) \int_{0}^{\infty} \mathrm{d} q \frac{q}{\kappa} e^{-2 \kappa z}\left(r_{2-}^{p}-r_{2-}^{s}\right)\left(e^{-2 \kappa \Delta z}-1\right)
$$


where the quantities $r_{2-}^{\sigma}$ must be computed for $\varepsilon_{2}=1$ and $\kappa_{2}=\kappa$. In other words, they are the reflection coefficients corresponding to the case where the half-space on the right-hand side of the wall is empty, and thus they agree with the reflection coefficients $r_{1-}^{\sigma}$ in equation (28). Obviously, equation (37) can be written in the form of equation (29) (with $d_{1} \mapsto z$ ), where the force density $f(z)$ can be derived, according to equation (30), from a potential $V(z)$ which now reads

$$
V(z)=-\frac{\hbar}{8 \pi^{2}} \int_{0}^{\infty} \mathrm{d} \xi \frac{\xi^{2}}{c^{2}}\left(\varepsilon_{2}-1\right) \int_{0}^{\infty} \mathrm{d} q \frac{q}{\kappa} e^{-2 \kappa z}\left(r_{2-}^{p}-r_{2-}^{s}\right)
$$

instead of equation (31).

Applying equation (32), we can again use equation (33) to introduce the force $F^{(\text {at })}(z)$ acting on an atom at position $z$ in the plate, where the potential from which the force can be derived is given by

$$
V^{(\mathrm{at})}(z)=-\frac{\hbar \mu_{0}}{8 \pi^{2}} \int_{0}^{\infty} \mathrm{d} \xi \xi^{2} \alpha(i \xi) \int_{0}^{\infty} \frac{\mathrm{d} q q}{\kappa}\left(r_{2-}^{p}-r_{2-}^{s}\right) e^{-2 \kappa z}
$$

instead of equation (34). Hence, equation (39) can be interpreted as the potential that a matter atom is subject to when the presence of the surrounding atoms of the (weakly dielectric) matter is taken into account, thus being the screened single-atom $\mathrm{CP}$ potential. It differs from the unscreened CP potential (2) (or, equivalently, (34)) in the $p$-polarization contributions. The result is in agreement with the one recently found in [18], in which equation (10) together with equation (13) is applied to a multi-plate cavity-like system and it is shown that the Casimir force acting on a plate embedded in such a system can be decomposed into two parts, where one part can be regarded as screened force.

\section{Discussion and Summary}

It is not surprising that the unscreened CP potential (equation (2) or, equivalently, equation (34)) must differ from the screened one (equation (39)), as can be seen from the respective physical meaning of the force (per transverse unit area) $\mathrm{d} F=f(z) \mathrm{d} z$ that acts on a slice of infinitesimal thickness $\mathrm{d} z$ (cf equation (29)). To obtain the unscreened $\mathrm{CP}$ force, a slice in the otherwise empty right half-space is considered (see figure 1(a)), leading - if equation (32) holds - to the force on a single atom in front of the wall.

In contrast, the screened CP force is obtained if the slice is unavoidably a part of the medium that fills the right half-space in figure 1(b), leading to the force on a medium atom. From a microscopical point of view, this force does not only result from the van der Waals forces between the medium atom under consideration and the atoms of the wall, but also between the medium atom and the other medium atoms. Since there are many more other medium atoms to the right rather than to the left of the medium atom under consideration, there will be a net effect, which is expected to diminish the force as compared to the single-atom case.

To illustrate this screening effect, let us compare the unscreened potential (equation (34)) and the screened potential (equation (39)) in the idealized limit where the wall 
can be regarded as a perfectly reflecting mirror such that $r_{1-}^{p}=1, r_{1-}^{s}=-1$. In this case equation (34) greatly simplifies (the expression in the square bracket equals -2 ), leading to the unscreened potential in the form of

$$
V^{(\mathrm{at})}(z)=-\frac{\hbar c}{64 \pi^{2} \varepsilon_{0}} \frac{1}{z^{4}} \int_{0}^{\infty} \mathrm{d} y \alpha\left(\frac{i c y}{2 z}\right) h(y),
$$

where the dimensionless function $h(y)$ is defined by

$$
h(y)=\int_{y}^{\infty} \mathrm{d} x x^{2} e^{-x}=\left(y^{2}+2 y+2\right) e^{-y} .
$$

In particular, in the large-distance limit equation (40) reduces to Casimir's and Polder's well-known formula [3] $\left[\int_{0}^{\infty} \mathrm{d} y h(y)=6\right]$

$$
V^{(\mathrm{at})}(z)=-\frac{3 \hbar c \alpha(0)}{32 \pi^{2} \varepsilon_{0}} \frac{1}{z^{4}} .
$$

Correspondingly, setting $r_{2-}^{p}=1$ and $r_{2-}^{s}=-1$ in equation (39), we may write the screened potential in the form of equation (40), where the function $h(y)$ changes to

$$
h(y)=y^{2} \int_{y}^{\infty} \mathrm{d} x e^{-x}=y^{2} e^{-y} .
$$

Since now $\int_{0}^{\infty} \mathrm{d} y h(y)=2$, it follows that in the large distance-limit the screened potential is one third of the unscreened one,

$$
V^{(\mathrm{at})}(z)=-\frac{\hbar c \alpha(0)}{32 \pi^{2} \varepsilon_{0}} \frac{1}{z^{4}}
$$

provided that $\alpha(0)$ is the same in both cases. The result clearly shows that the screening effect can be fairly large under certain conditions. In the short-distance limit, the asymptotic behaviour of the unscreened CP potential is commonly obtained by approximately setting $c \rightarrow \infty$, implying $\kappa \simeq q$ (non-retarded approximation), thus the leading term behaves like $z^{-3}$. It is not difficult to see that this term is missing in the screened potential, and that the same approximation would lead to a $z^{-1}$ distance law. It is, however, more than questionable whether this approximation is reasonable in the case of a medium atom. Moreover, when the atom can be no longer regarded as being surrounded by sufficiently many other atoms of the same kind, then the macroscopic description leading to equation (39) fails. Hence, even if the strict short-distance limit of Eq. (39) could be found by more elaborate methods, its meaning might be severly limited.

From the above we can conclude that electromagnetic vacuum forces acting on micro-objects that consist of collections of atomic constituents should be preferably calculated as Casimir forces, by assigning appropriately chosen permittivities and/or permeabilities to the micro-objects. This approach ensures that screening effects are properly taken into account. In particular, in the case of a weakly magnetodielectric object the total force can be obtained, to leading order, by superimposing screened $\mathrm{CP}$ forces acting on the atomic constituents. With increasing strength of the magnetodielectric properties, higher-order corrections (not considered in this paper) 
must be included in the calculation. This can be done in a systematic fashion, by starting from the exact formula for the Casimir force and expanding to higher powers in the electric and/or magnetic susceptibility.

In summary, we have studied relations between the Casimir force acting on a macroscopic body and the CP force acting on an atom, with special emphasis on planar structures. We have shown that the exact formula for the Casimir force contains as a special case the ordinary CP force acting on a single atom in front of a wall. Further, we have shown that the exact formula can also be used to calculate the CP force acting on an atom that is a constituent of bulk material that fills the half-space in front of the wall. In this case the surrounding atoms give rise to a screening effect that diminishes the $\mathrm{CP}$ force compared with the force that acts on an isolated atom.

\section{Acknowledgments}

We thank Stefan Scheel for discussions. CR is grateful for being granted a Thüringer Landesgraduiertenstipendium.

\section{References}

[1] London F 1930 Z. Phys. 63245

[2] London F 1937 Trans. Faraday Soc. 338

[3] Casimir H B G and Polder D 1948 Phys. Rev. 73360

[4] Milonni P W 1994 The Quantum Vacuum - An Introduction to Quantum Electrodynamics (San Diego: Academic Press, Inc.)

[5] Lifshitz E M 1955 Zh. Eksp. Teor. Fiz. 2994 (Sov Phys JETP 19562 73)

[6] Raabe C and Welsch D-G 2005 Phys. Rev. A 71013814

[7] McLachlan A D 1963 Proc. R. Soc. A 271 387; Mol. Phys. 7381

[8] Agarwal G S 1975 Phys. Rev. A 11243

[9] Wylie J M and Sipe J E 1984 Phys. Rev. A 301185

[10] Henkel C, Joulain K, Mulet J P and Greffet J J 2002 J. Opt. A: Pure Appl.Opt. 4109

[11] Buhmann S Y, Ho Trung Dung and Welsch D-G 2004 J. Opt B: Quantum Semiclass. Opt. 6 S127

[12] Knöll L, Scheel S and Welsch D-G 2001 Coherence and Statistics of Photons and Atoms ed J Per̆ina (New York: Wiley p 1) (arXiv:quant-ph/0006121)

[13] Kryszewski S 1993 Mol. Phys. 781225

[14] Buhmann S Y, Dung H T, Kampf T and Welsch D-G 2005 Eur. Phys. J. D 3515

[15] Landau L D and Lifschitz E M 1966 Lehrbuch der theoretischen Physik Bd. 5 - Statistische Physik (Berlin: Akademie Verlag)

[16] Tomaš M S 1995 Phys. Rev. A 512545

[17] Chew W C 1995 Waves and Fields in Inhomogeneous Media (New York: IEEE Press)

[18] Tomaš M S 2005 Phys. Rev. A 71 060101(R), arXiv:quant-ph/0502145, quant-ph/0505127 